\title{
Polydimethylsiloxane based microfluidic diode
}

\author{
M L Adams ${ }^{1}$, M L Johnston ${ }^{2}$, A Scherer ${ }^{2}$ and S R Quake ${ }^{3}$ \\ ${ }^{1}$ ENSCO, Inc, Melbourne, FL 32940, USA \\ ${ }^{2}$ California Institute of Technology, Pasadena, CA 91125, USA \\ ${ }^{3}$ Stanford University, Stanford, CA 94305, USA \\ E-mail: adams.mark@ensco.com
}

Received 18 March 2005, in final form 18 May 2005

Published 14 June 2005

Online at stacks.iop.org/JMM/15/1517

\begin{abstract}
In this paper, we present a novel elastomer-based microfluidic device for rectifying flow. The device is analogous to an electronic diode in function since it allows flow in one direction and stops flow in the opposing direction. The device is planar, in-line and can be replica molded via standard soft lithography techniques. The fabrication process is outlined in detail and follows a simple procedure that requires only photolithography and one replica molding step. Several geometries of devices are presented along with their flow versus pressure characteristics. A brief discussion of the device behavior is presented along with possible uses for the device.
\end{abstract}

(Some figures in this article are in colour only in the electronic version)

\section{Introduction}

Microfluidic technology has revolutionized lab-on-a-chip applications during the past decade [1-5]. However, the process of creating a suitable valve system is often problematic. In many applications, a one-way or check valve becomes necessary to safely perform tasks such as drug delivery where backflow must be prevented. The microfluidic diode provides a unique solution to this problem and is analogous to an electronic diode in function. Where an electronic diode prevents current flow in one direction, the fluidic diode prevents fluid flow in a certain direction and serves the function of a rectifier. Although there have been other attempts at making valves which limit flow in one direction, they almost always involve a very complex fabrication procedure in which many steps are needed to produce the device [6-11]. The fluidic diode is a singlelayer, planar device that can be fully integrated with standard multilayer soft lithography technology.

The applications for such a device are many and varied. One of the most important uses of the device is to prevent backflow. In many situations, it is desirable to avoid a solution leaking back from where it had come. Another use is in the creation of microfluidic logic. Once again, an analogy can be drawn from diode-diode logic in electronics. The fluidic diode can be a basic building block for logic devices such as fluidic inverters and nand/nor gates. We have envisioned making pumps, pressure reservoirs or oscillators with the fluidic diode.

\section{Design}

The device is based upon a mechanical effect in which a flap of polydimethylsiloxane (PDMS) seals to a channel in one direction and opens in the flow direction. This is very similar to a door which has a door jam on one side but is free to swing in the other direction. The device is illustrated in figure 1. It is important to note that the flap hangs from the top of the wider channel. This channel is also higher than the other channel, so that the flap can completely seal against the small channel. The properties of the diode can be adjusted by changing the flap thickness as well as the ratio of channel heights. For most applications, the channel height ratio needs to be at least 2:1 and the ratio between the large channel height and the flap thickness should be 3:1 minimum or the flap will be too stiff. The distance between the flap and the channel orifice is proportional to the flap thickness but should be minimized to improve performance. The channel containing the flap had a maximum width of $100 \mu \mathrm{m}$ and a minimum width of $50 \mu \mathrm{m}$ at the channel juncture. The shorter channel had a maximum width of $50 \mu \mathrm{m}$ and narrowed to $25 \mu \mathrm{m}$ at the channel juncture. The flap thickness varied with the channel height and is discussed below. Typical diode parameters are illustrated in table 1 . 


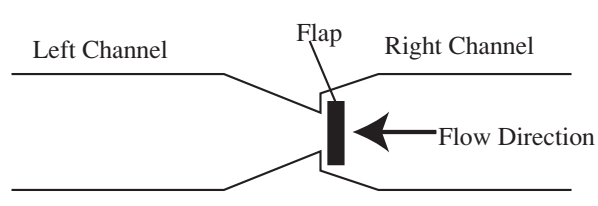

Top View

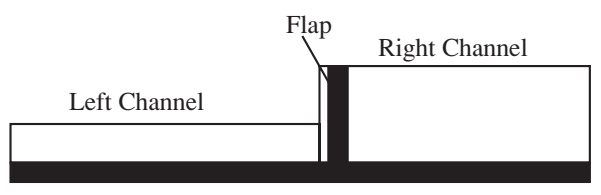

Side View

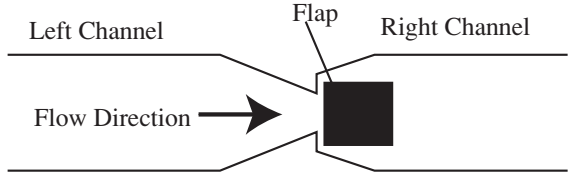

Top View

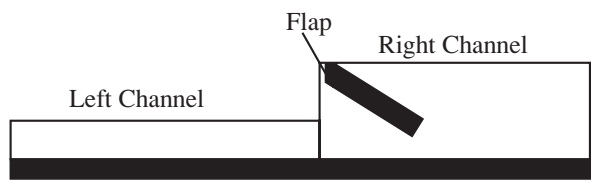

Side View

(a) Closed Position

(b) Open Position

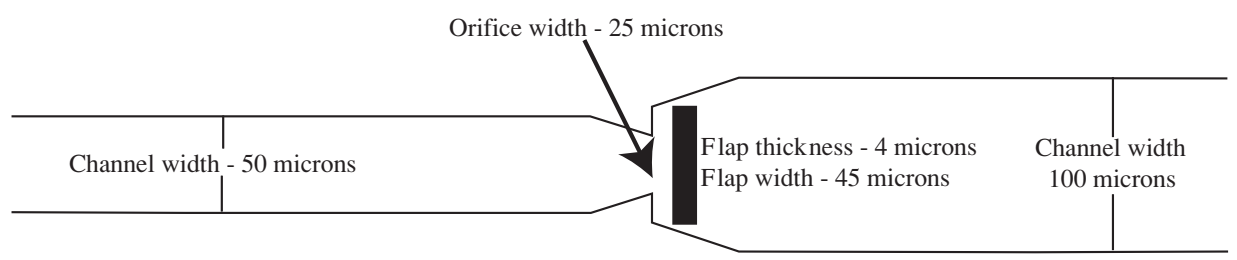

Top View

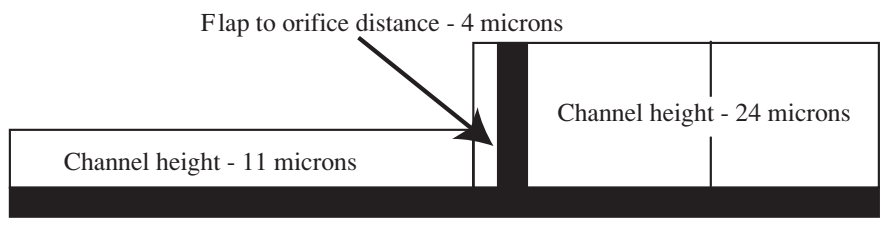

Side View

(c) Typical Dimensions - Drawing not to scale

Figure 1. Microfluidic diode illustration. The flow from the right direction is stopped as the flap presses against the orifice of the left channel. Flow from the left is allowed to flow since the flap is unobstructed in the right channel. $(a)$ illustrates the flap in the closed position. (b) illustrates the flap in the open position. (c) A typical fluidic diode's dimensions.

Table 1. Typical diode parameters.

\begin{tabular}{llllll}
\hline $\begin{array}{l}\text { Aspect ratio } \\
\text { (approximate) }\end{array}$ & $\begin{array}{l}\text { Channel height } \\
(\mu \mathrm{m})\end{array}$ & $\begin{array}{l}\text { Flap height } \\
(\mu \mathrm{m})\end{array}$ & $\begin{array}{l}\text { Flap width } \\
(\mu \mathrm{m})\end{array}$ & $\begin{array}{l}\text { Flap thickness } \\
(\mu \mathrm{m})\end{array}$ & $\begin{array}{l}\text { Orifice-flap } \\
\text { distance }(\mu \mathrm{m})\end{array}$ \\
\hline $2: 1$ & 11 & 24 & 45 & 4 & 4 \\
$3: 1$ & 11 & 33 & 45 & 6 & 5 \\
$4: 1$ & 11 & 42 & 45 & 7 & 5 \\
\hline
\end{tabular}

\section{Fabrication}

A typical fluidic diode is fabricated through standard replica molding techniques. A mold is created through a bilayer photoresist process. The aspect ratios of the device are controlled by changing the photoresist type. The mold is created using two different types of Microchem SU8 2000 series photoresist. For example, a 2:1 aspect ratio device would consist of a layer of SU8-2010 and SU8-2015. The SU8-2010 is spun onto a clean silicon wafer using a Laurel spinner. The resist is spread onto the wafer at 500 RPM for $8 \mathrm{~s}$ and then ramped up to $5000 \mathrm{RPM}$ at an acceleration of $340 \mathrm{RPM} \mathrm{s}^{-1}$. Once $5000 \mathrm{RPM}$ has been reached, the sample is spun for $30 \mathrm{~s}$. The wafer is then soft baked on two separate hotplates set at $65^{\circ} \mathrm{C}$ and $95{ }^{\circ} \mathrm{C}$ for $1 \mathrm{~min}$ at $65^{\circ} \mathrm{C}, 2 \mathrm{~min}$ at $95^{\circ} \mathrm{C}$ and $1 \mathrm{~min}$ at $65^{\circ} \mathrm{C}$. After soft baking, the wafer is exposed for $10 \mathrm{~s}$ at $5.5 \mathrm{~mW} \mathrm{~cm} \mathrm{~cm}^{-2}$ using a Karl-Suss MJB3 mask aligner. The wafer is then post exposure baked (PEB) for $1 \mathrm{~min}$ at $65^{\circ} \mathrm{C}, 2 \mathrm{~min}$ at $95^{\circ} \mathrm{C}$ and $1 \mathrm{~min}$ at $65^{\circ} \mathrm{C}$ on the two hotplates. The sample is then allowed to cool to room temperature and then developed in Microchem SU8 developer for $2 \mathrm{~min}$. Once developed the sample is blown dry with nitrogen.

After drying, the sample is ready for the next layer of SU8 to be applied. The SU8-2015 is applied in a similar fashion directly on top of the SU8-2010. The resist is spread onto the wafer at 500 RPM for $8 \mathrm{~s}$ and then ramped up to $1700 \mathrm{RPM}$ at an acceleration of $340 \mathrm{RPM} \mathrm{s}^{-1}$. Once $1700 \mathrm{RPM}$ has been reached, the sample is spun for $30 \mathrm{~s}$. The wafer is then soft baked on two separate hotplates set at $65{ }^{\circ} \mathrm{C}$ and $95{ }^{\circ} \mathrm{C}$ for 


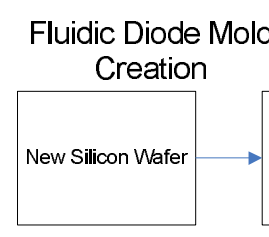

Layer 1

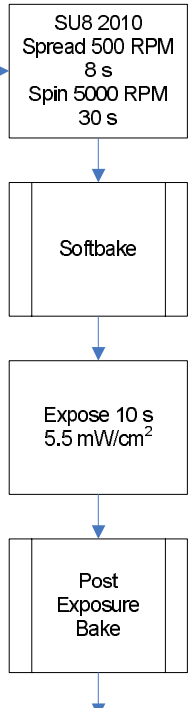

Layer 2

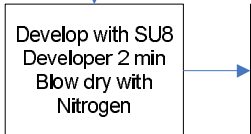

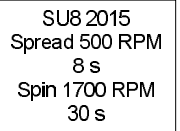
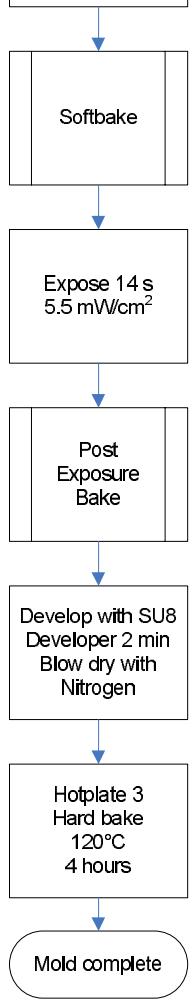

Predefined Processes
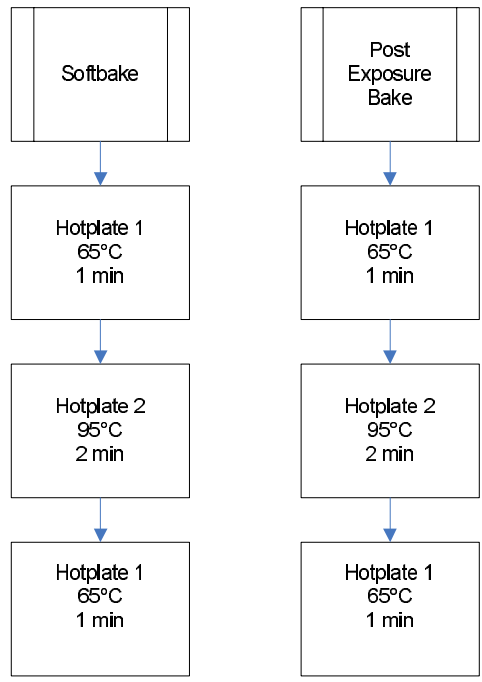

Fluidic Diode
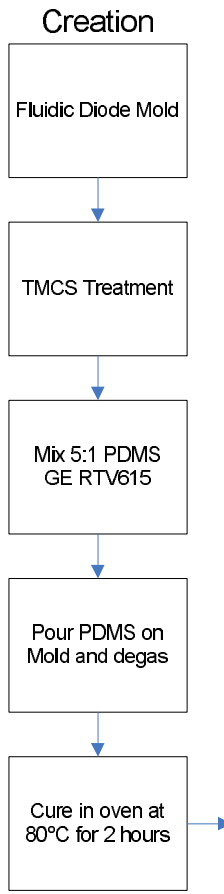

emove diode from mold and al to glass with ethanol

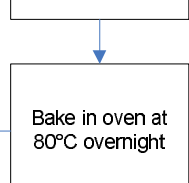

Figure 2. Fabrication process flow for a 2:1 aspect ratio microfluidic diode.

2 min at $65{ }^{\circ} \mathrm{C}, 4 \mathrm{~min}$ at $95{ }^{\circ} \mathrm{C}$ and $1 \mathrm{~min}$ at $65^{\circ} \mathrm{C}$. After soft baking, the wafer is exposed for $14 \mathrm{~s}$ at $5.5 \mathrm{~mW} \mathrm{~cm} \mathrm{~cm}^{-2}$ using a Karl-Suss MJB3 mask aligner. The wafer is then post exposure baked for $1 \mathrm{~min}$ at $65^{\circ} \mathrm{C}, 2 \mathrm{~min}$ at $95^{\circ} \mathrm{C}$ and $1 \mathrm{~min}$ at $65^{\circ} \mathrm{C}$ on the two hotplates. The sample is then allowed to cool to room temperature and then developed in Microchem SU8 developer for $2 \mathrm{~min}$. Once developed the sample is blown dry with nitrogen. After drying, the sample is hard baked on a hotplate at $120^{\circ} \mathrm{C}$ for $4 \mathrm{~h}$. The mold is ready for replication after the hard bake has completed. The above process yields channel heights of approximately 11 and $24 \mu \mathrm{m}$ for the two channels which is slightly greater than the $2: 1$ aspect ratio required.

The microfluidic diode device is created by coating the mold with PDMS which typically involves a bulk elastomer (part A) and a curing agent (part B). GE RTV615 is used with 


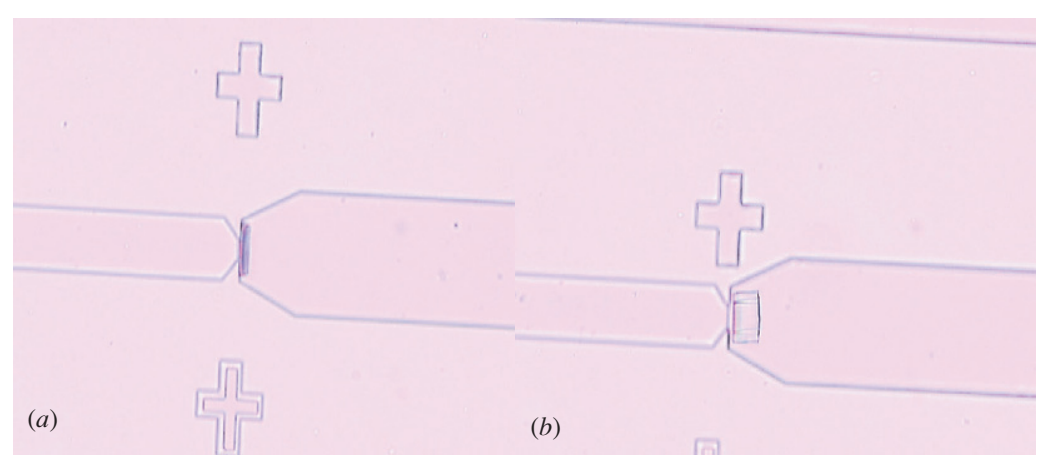

Figure 3. (a) Fluidic diode with the flap rolled upon itself. (b) The flap was unrolled into the channel.

a 5:1 ratio of part A to part B. The stiffness of the flap can be controlled by changing the ratio or using a stiffer PDMS such as Dow Corning Sylgard ${ }^{\mathrm{TM}}$. The mold is treated with trimethylchlorosilane and then the PDMS is mixed and poured onto the mold. The PDMS is then evacuated to assure that no air bubbles will interfere with the diode operation. Once evacuated, the mold is placed into an $80^{\circ} \mathrm{C}$ oven for $2 \mathrm{~h}$ to cure the PDMS. After the device has cured, it is removed from the mold and holes are punched into the flow channels to allow fluid entry. The final device is then sealed to a glass slide using ethanol and baked in an $80{ }^{\circ} \mathrm{C}$ oven overnight. The ethanol provides a better seal to the glass and increases the delamination pressure to $25 \mathrm{psi}(172.4 \mathrm{kPa})$. Experiments have shown that without the ethanol treatment the PDMS delaminates from the glass at $15 \mathrm{psi}(103.4 \mathrm{kPa})$. For all of the devices tested, the channel and flap widths were held constant. The entire process flow is illustrated in figure 2 .

\section{Results and discussion}

One potential problem that can occur during fabrication involves the deformation of the flap. Since the flap is quite tall and narrow, when the elastomeric device is removed from the mold, the flap is placed under substantial tension. Once free from the mold, the flap can snap back and sometimes, depending upon the aspect ratio, the flap can roll up onto itself. This causes a partial blockage within the channel which limits, but does not prevent flow. A device in which the flap is rolled upon itself is shown in figures $3(a)$ and $(b)$ which illustrates the flap in its unrolled state.

By changing the flap parameters, one can adjust the turnon pressure of the diode as well as the response time of the flap. The stiffer the flap, the faster it will respond when the flow direction is reversed. Devices with different geometries were characterized by applying regulated pressure to the flow direction and then the non-flow direction. Each device was tested with $100 \mu \mathrm{M}$ fluorescein with an inverted microscope and mercury lamp. A picture of the tested device appears in figure 4 . The device shown has two microfluidic diodes which are facing in opposing directions. The fluorescein enabled the easy determination of when the fluid started flowing as well as if there was any leakage in the non-flow direction. Table 2 lists turn-on pressure and reverse standoff pressure for some devices. As can be seen, the reverse standoff is quite high and generally the sample became unsealed from the glass substrate before the device failed.
Table 2. Microfluidic diode performance as a function of geometry.

\begin{tabular}{lll}
\hline $\begin{array}{l}\text { Aspect ratio } \\
\text { (height/thickness) }\end{array}$ & Turn on—psi (kPa) & $\begin{array}{l}\text { Reverse standoff—psi } \\
(\mathrm{kPa})\end{array}$ \\
\hline $2: 1$ & $8.3(57.2)$ & $25(172.4)$ \\
$3: 1$ & $5.5(37.9)$ & $25(172.4)$ \\
$4: 1$ & $3.2(22.1)$ & $25(172.4)$ \\
\hline
\end{tabular}

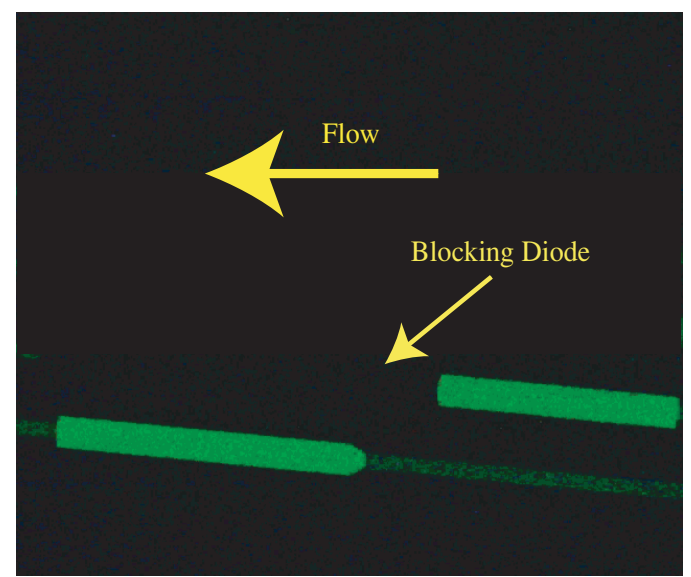

Figure 4. Diodes tested with $100 \mu \mathrm{M}$ fluorescein. The fluorescein flows in the direction of flow for the bottom diode and does not flow in the opposing device.

Since the fluidic diode is an obvious analog of the electronic diode, it was of interest to try and determine the analogy of an $I-V$ curve. Since in microfluidics pressure is equivalent to voltage, much of the data had already been acquired. However, the fluidic equivalent to current is flow which is very difficult to measure in a microfluidic system. The flow rate can be calculated as follows given the pressure drop and the fluidic resistance of the channel; however, experimental data were needed to verify the calculations. Given laminar flow conditions and a Newtonian fluid, the volume flow rate of a rectangular channel is given by

$$
Q=\Delta P / R
$$

where $\Delta P$ is the pressure difference and $R$ is the fluidic resistance. In order to determine an approximate flow rate, fluorescent beads were used to track the flow inside the channel. Since the exact length of the channel was known, the speed of the bead could be determined by measuring the distance traveled in a certain time period. Through this method 
Flow rate vs. Pressure

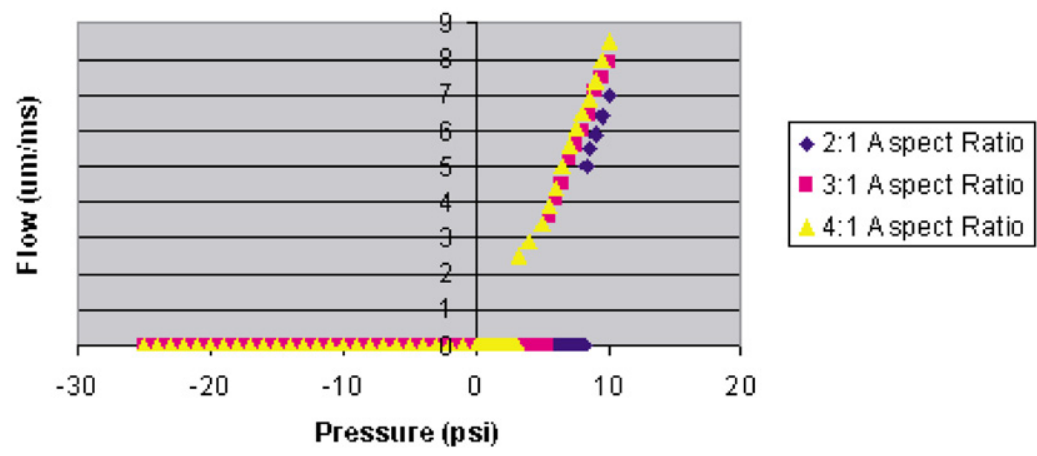

Figure 5. A flow rate versus pressure curve for the three fluidic diode geometries described above. This curve is analogous to an electronic diode's $I-V$ curve. The height, thickness and stickiness of the flap control the turn-on pressure.

a $Q_{1}-P$ (flow rate versus pressure) curve was generated and is shown in figure 5. It can be seen that the higher aspect ratio channels achieve slightly larger flow rates for a given pressure. This can be attributed to the dependence of fluidic resistance on channel geometry as described in equation (1.2)

$$
R=\frac{12 \mu L}{h w^{3}}
$$

where $L, w$ and $h$ are the length, width and height of the channel, respectively and $\mu$ is the viscosity. For a rectangular channel, $w$ and $h$ are interchangeable. Thus, the fluidic resistance simply assumes a high aspect ratio [12].

The turn-on or actuation pressure is a function of the flap parameters and is also affected by the stickiness of the flap to the channel orifice and channel bottom. However, since the diode performs well over a range of pressures and geometries, the stickiness was determined not to be critical. The data presented in figure 5 were acquired with a 0.1 psi $(689.5 \mathrm{~Pa})$ accuracy pressure regulator and a video camera to capture the fluorescent beads flowing within the channel. With a more accurate pressure regulator and a faster video capture mechanism, the curves could be filled in with greater detail.

\section{Conclusion}

We have presented a novel microfluidic device which can make a tremendous impact in the area of microfluidics. Simply by changing the geometry of the device the actuation pressure and flow rate can be controlled. The microfluidic diode is a simple, functional device which can form the basis of a vast number of devices such as microfluidic logic, fluidic oscillators, pumps and check valves. The device shows a strong analogy to the electronic diode and since it is planar and in-line, the microfluidic diode can be fully utilized through large scale integration.

\section{References}

[1] Xi Y N et al 1996 Complex optical surfaces formed by replica molding against elastomeric masters Science 273 347-9

[2] Xia Y N and Whitesides G M 1998 Soft lithography Angew. Chem., Int. Ed. Engl. 37 550-75

[3] Fu A Y et al 1999 A microfabricated fluorescence-activated cell sorter Nat. Biotechnol. 17 1109-11

[4] Unger M A et al 2000 Monolithic microfabricated valves and pumps by multilayer soft lithography Science $\mathbf{2 8 8} 113-6$

[5] Chou $\mathrm{H}$ et al 1999 A microfabricated device for sizing and sorting DNA molecules Proc. Natl Acad. Sci. USA 96 11-3

[6] Shoji S et al 1992 A study of a high-pressure micropump for integrated chemical analyzing systems Sensors Actuators A 32 335-9

[7] Seidemann V, Butefisch S and Buttgenbach S 2002 Fabrication and investigation of in-plane compliant SU8 structures for MEMS and their application to micro valves and micro grippers Sensors Actuators A 97 457-61

[8] Seidemann V et al 2002 SU8-micromechanical structures with in-situ fabricated movable parts Microsyst. Technol. 8 348-50

[9] Hasselbrink E F, Shepodd T J and Rehm J E 2002 High-pressure microfluidic control in lab-on-a-chip devices using mobile polymer monoliths Anal. Chem. 74 4913-8

[10] Sim W Y et al 2003 A phase-change type micropump with aluminum flap valves J. Micromech. Microeng. 13 286-94

[11] Hosokawa K and Maeda R 2000 A pneumatically-actuated three-way microvalve fabricated with polydimethylsiloxane using the membrane transfer technique J. Micromech. Microeng. 10 415-20

[12] Kovacs G 1998 Micromachine Transducers Sourcebook (Palo Alto, CA: WCB/McGraw-Hill) p 911 